Bopp operators and phase-space spin dynamics: application to rotational quantum Brownian motion

This article has been downloaded from IOPscience. Please scroll down to see the full text article.

2007 J. Phys. A: Math. Theor. 404635

(http://iopscience.iop.org/1751-8121/40/17/015)

View the table of contents for this issue, or go to the journal homepage for more

Download details:

IP Address: 155.210.93.57

The article was downloaded on $25 / 06 / 2010$ at $14: 43$

Please note that terms and conditions apply. 


\title{
Bopp operators and phase-space spin dynamics: application to rotational quantum Brownian motion
}

\author{
D Zueco $^{1}$ and I Calvo ${ }^{2}$ \\ ${ }^{1}$ Departamento de Física de la Materia Condensada e, Instituto de Ciencia de Materiales de \\ Aragón, C.S.I.C.-Universidad de Zaragoza, E-50009 Zaragoza, Spain \\ ${ }^{2}$ Laboratorio Nacional de Fusión, Asociación EURATOM-CIEMAT, E-28040 Madrid, Spain \\ E-mail: zueco@unizar.es and ivan.calvo@ciemat.es
}

Received 23 November 2006, in final form 3 March 2007

Published 11 April 2007

Online at stacks.iop.org/JPhysA/40/4635

\begin{abstract}
For non-relativistic spinless particles, Bopp operators give an elegant and simple way to compute the dynamics of quasiprobability distributions in the phase-space formulation of Quantum Mechanics. In this work, we present a generalization of Bopp operators for spins and apply our results to the case of open spin systems. This approach allows to take the classical limit in a transparent way, recovering the corresponding Fokker-Planck equation.
\end{abstract}

PACS numbers: 03.65.Sq, 03.65.Vf, 03.65.Yz, 05.40.-a

\section{Introduction}

The seed of the phase-space formulation of Quantum Mechanics dates back to 1932, when Wigner introduced his famous quasi-probability function in phase space [1]:

$$
W_{\psi}(q, p)=\frac{1}{2 \pi \hbar} \int_{-\infty}^{\infty} \mathrm{d} u \psi^{*}\left(q-\frac{1}{2} u\right) \psi\left(q+\frac{1}{2} u\right) \mathrm{e}^{-\mathrm{i} u p / \hbar}
$$

The relation between Wigner's function and Weyl's correspondence was understood by Groenewold [2] and fully developed by Moyal [3], who established an independent formulation of Quantum Mechanics in phase space for the canonical Poisson bracket $\{q, p\}=1$. In this equivalent, 'statistical' or hydrodynamical formulation of Quantum Mechanics, the expectation value of an operator is computed as the average of the corresponding function on the phase space with the 'probability' density given by the Wigner function.

In light of these results, it was suggestive to look for an autonomous formulation of Quantum Mechanics in phase space involving only classical functions and valid for any Poisson bracket. This is the so-called deformation quantization programme proposed by M Flato and collaborators in the 1970s [4]. The problem is stated as follows: given a smooth 
manifold $M$ with Poisson bracket $\{\cdot, \cdot\}: C^{\infty}(M) \times C^{\infty}(M) \rightarrow C^{\infty}(M)$, find an $\mathbb{R}[[\hbar]]$-bilinear associative deformation of the point-wise product on $C^{\infty}(M)$, , such that

$$
f \star g-g \star f=\hbar\{f, g\}+O\left(\hbar^{2}\right), \quad \forall f, g \in C^{\infty}(M) .
$$

The existence of $\star$-products for any Poisson manifold was a longstanding problem in Mathematics solved in 1997 by M Kontsevich [5]. As a consequence of his more general Formality Theorem, he showed that any Poisson manifold can be quantized by deformation and classified the $\star$-products (see globalization aspects in [6]).

One of the main advantages of the deformation quantization prescription (or equivalently, the phase-space formulation of Quantum Mechanics) resides in the quantum-classical transition problem. The classical limit is obtained in a clear and mathematically rigorous way by taking $\hbar \rightarrow 0$.

The phase-space formalism has been applied successfully to the description of a spinless particle [7, 8], whose phase space is $\mathbb{R}^{2}$ with the canonical Poisson bracket. More complicated is the phase-space description of a spin, the phase space being the sphere $S^{2}$. The phase space of a spin was given in terms of the atomic (or spin) coherent states, [9-12], which in a sense generalizes the Cahill-Glauber construction for the spinless case [13].

Although the results of Kontsevich guarantee the existence of a $\star$-product for any classical system (i.e., for any Poisson manifold), his explicit formula is rather complicated and, for example, it is not obvious how to take advantage of the symmetries of the system. Várilly and Gracia-Bondía (see [14] for $S U(2)$ and [15] for any compact group) showed that the appropriate set-up for systems with symmetries is given by the Stratonovich-Weyl (SW) correspondence [16] (see section 2). In addition, they proved that this approach is equivalent to the spin coherent state representation. These works were generalized by Brif and Mann $[17,18]$, establishing the SW correspondence for systems with an arbitrary finite-dimensional Lie-group symmetry.

In the present work, we tackle the problem of the dynamics of spin systems in the phase-space formulation and its classical limit. Recently Klimov and Espinoza [19] derived a differential form for the star product in the spin case. On the other hand, in the spinless case the evaluation of the star product becomes simpler with the use of Bopp operators [20]. We use Klimov and Espinoza's results and work out the Bopp operators for spins. Takahashi and Shibata already found this generalization for the specific cases of normal and anti-normal ordering [10, 11]. Herein, we re-obtain their results and generalize them for an arbitrary ordering. In particular, we obtain the Bopp operators for the important case of symmetric ordering. We will apply our results to show how the use of Bopp operators simplifies the derivation of the dynamical equations for spins.

Finally, we deal with the problem of a system in contact with a bosonizable bath as a model of quantum dissipation [21]. Classically, the effective dynamics of the system is described by Langevin or Fokker-Planck equations [22]. In the quantum domain and under certain conditions (essentially weak coupling between system and environment), the dynamics of the system can be formulated in terms of a quantum master equation for the (reduced) density matrix [23]. For spinless particles the phase-space transform of the master equations yields the quantum generalization of the Klein-Kramers equations [24]. In the sense of Caldeira and Leggett, who pose the open system dynamics as a quantization problem [25], the phase-space formulation 'closes the circle', giving a quantum version of the Fokker-Planck equations. With the help of the Bopp operators for spins we easily obtain quantum Fokker-Planck equations, recovering the corresponding classical Fokker-Planck equations for the rotational Brownian motion [26] in the limit $\hbar \rightarrow 0$. This provides a natural framework to link the classical and quantum theories of dissipation. 
The paper is organized as follows:

Section 2 is a brief survey on the phase-space formulation of Quantum Mechanics from the point of view of the Stratonovich-Weyl postulates. The general results are illustrated by the example of the non-relativistic spinless particle, introducing the definition of Bopp operators.

Section 3 deals with the phase-space formalism of spin systems. We generalize the Bopp operators and tackle the problem of the quantum-classical transition in these systems. In addition, we work out in detail the particular case of quadratic Hamiltonians.

In section 4 , we apply our results to open quantum spin systems. We transform into phase space the density matrix equation for a spin in contact with a thermal bath, obtaining quantum Fokker-Planck equations. This transformation becomes simple with the help of Bopp operators. We write explicitly the dissipative equations for the linear Hamiltonian (isotropic spin) and compare it with its spinless analogue, the damped harmonic oscillator. Finally, we take the classical limit for a general quantum Fokker-Planck equation, recovering the corresponding classical equation.

\section{Phase-space Quantum Mechanics}

In this section, we review some basic facts and results on phase-space quantization. The material is now standard and we closely follow the conventions and notation of $[17,18]$.

\subsection{The $S W$ postulates}

Consider a physical system possessing a group of symmetries described by a finitedimensional, connected and simply connected Lie group $G$. We denote the phase space of the system by $\mathcal{P}$ and assume that $G$ acts transitively on $\mathcal{P}$. That is, for any $x_{1}, x_{2} \in \mathcal{P}$, there exists $g \in G$ such that $g \cdot x_{1}=x_{2}$. Let $\mathcal{H}$ be the Hilbert space of our system, $\mathcal{O}(\mathcal{H})$ the set of operators on $\mathcal{H}$ and $\mathcal{U}: G \rightarrow \mathcal{O}(\mathcal{H})$ an irreducible unitary representation of $G$. The Stratonovich-Weyl correspondence ([16]) is a $\sigma$-parameterized map $W^{(\sigma)}: \mathcal{O}(\mathcal{H}) \rightarrow \mathcal{C}^{\infty}(\mathcal{P})$ satisfying the following properties for any $A, B \in \mathcal{O}(\mathcal{H})$.

(i) Linearity: $A \mapsto W_{A}^{(\sigma)}$ is linear and bijective.

(ii) Reality: $W_{A^{\dagger}}^{(\sigma)}(x)=W_{A}^{(\sigma)}(x)^{*}$, where $A^{\dagger}$ is the adjoint of $A$.

(iii) Standardization:

$$
\int_{\mathcal{P}} W_{A}^{(\sigma)}(x) \mathrm{d} \mu(x)=\operatorname{Tr} A .
$$

(iv) Traciality:

$$
\int_{\mathcal{P}} W_{A}^{(\sigma)}(x) W_{B}^{(-\sigma)}(x) \mathrm{d} \mu(x)=\operatorname{Tr}(A B) .
$$

(v) Covariance:

$$
W_{A^{g}}^{(\sigma)}(x)=W_{A}^{(\sigma)}(g \cdot x), \quad \forall g \in G,
$$

where $\mu$ is an invariant measure and $A^{g}:=\mathcal{U}\left(g^{-1}\right) A \mathcal{U}(g)$.

As proved by Wigner et al [7] for spinless particles, by Várilly and Gracia-Bondía for spins [14] and recently by Brif and Mann [17, 18] for systems with an arbitrary Lie-group symmetry, properties (i)-(v) uniquely determine the SW correspondence.

Reality condition (ii) ensures that $W_{A}^{(\sigma)}$ is real whenever $A$ is Hermitian and property (iii) is simply a normalization. Linearity and Traciality conditions are essential to the formal 
interpretation of Quantum Mechanics as a statistical theory. Let $W_{\varrho}^{(\sigma)}$ be the image by the SW correspondence of the density matrix of the system, $\varrho$. For any operator $A,\langle A\rangle=\operatorname{Tr}(A \varrho)$ and due to (iv) we obtain

$$
\langle A\rangle=\int_{\mathcal{P}} W_{A}^{(\sigma)}(x) W_{\varrho}^{(-\sigma)}(x) \mathrm{d} \mu(x) .
$$

Therefore, the expectation value of an observable $A$ can be computed as the average on $\mathcal{P}$ of $W_{A}^{(\sigma)}$ weighted by $W_{\varrho}^{(-\sigma)}$. However, $W_{\varrho}^{(\sigma)}$ may be negative at some points ([7]), so that in general it does not define a true probability distribution. For this reason $W_{\varrho}^{(\sigma)}$ is sometimes called a quasiprobability distribution.

Finally, the covariance property (v) means that the SW correspondence commutes with the action of $G$, thus preserving the symmetry.

Note that under the SW correspondence, an operator is mapped to a set of functions labelled by $\sigma$, which is related to the operator ordering prescription. In particular, $W_{A}^{(\sigma)}$ for $\sigma=0,1$ and -1 are known, respectively, as symmetric, normal and antinormal functions. The nomenclature is due to the fact that, for the spinless particle, $\sigma=0$ corresponds to the symmetric ordering ([3]), whereas $\sigma=1,-1$ are associated with the normal and antinormal orderings, respectively [27].

\subsection{The SW kernel}

The vector space $\mathcal{O}(\mathcal{H})$ equipped with the bilinear form $(A, B)=\operatorname{Tr}\left(A^{\dagger} B\right)$ is a Hilbert space and due to the Riesz theorem, there exists a $\sigma$-parameterized set of operator-valued functions on $\mathcal{P}, \Delta^{(\sigma)} \in C^{\infty}(\mathcal{P}) \otimes \mathcal{O}(\mathcal{H})$, called the SW kernels, such that

$$
W_{A}^{(\sigma)}(x)=\operatorname{Tr}\left(A \Delta^{(\sigma)}(x)\right)
$$

Properties (ii)-(v) introduced above are equivalent to the following conditions on the kernels $([14,17,18])$ :

(ii') $\Delta^{(\sigma)}(x)=\Delta^{(\sigma)}(x)^{\dagger}$

(iii') $\int_{\mathcal{P}} \Delta^{(\sigma)}(x) \mathrm{d} \mu(x)=1$

$\left(\mathrm{iv}^{\prime}\right) \Delta^{(\sigma)}(x)=\int_{\mathcal{P}} \operatorname{Tr}\left(\Delta^{(\sigma)}(x) \Delta^{(-\sigma)}\left(x^{\prime}\right)\right) \Delta^{(\sigma)}\left(x^{\prime}\right) \mathrm{d} \mu\left(x^{\prime}\right)$

$\left(\mathrm{v}^{\prime}\right) \Delta^{(\sigma)}(g \cdot x)=\Delta^{(\sigma)}(x)^{g^{-1}}, \quad \forall g \in G$.

Remarkably, the tracial property (iv') allows us to invert the SW map (2), yielding a generalization of the Weyl rule:

$$
A=\int_{\mathcal{P}} W_{A}^{(\sigma)}(x) \Delta^{(-\sigma)}(x) \mathrm{d} \mu(x) .
$$

Note that the kernel involved in (2) is $\Delta^{(\sigma)}$, whereas the kernel entering formula (3) is $\Delta^{(-\sigma)}$. The case $\sigma=0$ is privileged and $\Delta^{(0)}$ is said to be a self-dual kernel.

\subsection{The star product}

Once the Stratonovich-Weyl correspondence has been constructed, the connection with the notion of $\star$-product is straightforward. The $\star$-product is obtained by transferring to $\mathcal{P}$ the associative algebra structure of $\mathcal{O}(\mathcal{H})$ through the SW map. That is,

$$
\left(W_{A}^{(\sigma)} \star W_{B}^{(\sigma)}\right)(x):=W_{A B}^{(\sigma)}(x),
$$

for any two operators $A$ and $B .^{3}$

3 A more general relation mixing different orderings can be given (see, e.g., [18]), but we will not discuss it in this work. 
At this point, a natural question arises. Given an arbitrary $\star$-product on $C^{\infty}(\mathcal{P})$, is there a Poisson bracket associated with it in a canonical way? The answer is positive and the proof is easy. Using associativity, a direct computation shows that

$$
\{f, g\}=\frac{f \star g-g \star f}{\hbar} \bmod \hbar, \quad f, g \in C^{\infty}(\mathcal{P})
$$

is a Poisson bracket. This is a nice way to see that any quantum system has a corresponding classical system which is obtained in the limit $\hbar \rightarrow 0$ by replacing commutators by Poisson brackets. For example, we can write the transformation of the von Neumann equation to phase space:

$\partial_{t} \varrho=-\frac{\mathrm{i}}{\hbar}[H, \varrho] \longmapsto \partial_{t} W_{\varrho}^{(\sigma)}=-\frac{\mathrm{i}}{\hbar}\left(W_{H}^{(\sigma)} \star W_{\varrho}^{(\sigma)}-W_{\varrho}^{(\sigma)} \star W_{H}^{(\sigma)}\right)$

where $H$ is the Hamiltonian of the system. Hence, the star product determines the dynamics on the phase space and reproduces the classical Hamilton equations in the limit $\hbar \rightarrow 0$.

\subsection{An example: the spinless non-relativistic particle}

The classical phase space of the spinless non-relativistic particle is $\mathbb{R}^{2}$ with canonical Poisson bracket in coordinates $(q, p)$ :

$$
\{q, p\}=1 \text {. }
$$

The Hilbert space of the quantum system is $L^{2}(\mathbb{R})$ and its dynamical symmetry group is the Heisenberg-Weyl group $H_{3}$. The Lie algebra of $H_{3}$ is generated by three elements $I, a$ and $a^{\dagger}$ with Lie brackets:

$$
[a, I]=\left[a^{\dagger}, I\right]=0, \quad\left[a, a^{\dagger}\right]=I .
$$

i.e. $a^{\dagger}$ and $a$ are creation and annihilation bosonic operators, respectively.

The SW correspondence in this case maps each operator $A(\hat{q}, \hat{p})$ into $C^{\infty}\left(\mathbb{R}^{2}\right)$, where

$$
(\hat{q} \psi)\left(q_{0}\right):=q_{0} \psi\left(q_{0}\right), \quad(\hat{p} \psi)\left(q_{0}\right):=-\left.\mathrm{i} \hbar \frac{\partial \psi}{\partial q}\right|_{q_{0}}, \quad \forall q_{0} \in \mathbb{R}
$$

for any $\psi \in L^{2}(\mathbb{R})$.

Using the standard complex coordinate $z=(q+\mathrm{i} p) / \sqrt{2 \hbar}$ the SW kernel reads

$$
\Delta^{(\sigma)}(z)=1 / \pi \int_{\mathbb{C}} \mathrm{e}^{\frac{\sigma}{2}|\xi|^{2}} \mathrm{e}^{\xi^{*} z-\xi z^{*}} \mathrm{e}^{\xi a^{+}-\xi^{*} a} \mathrm{~d}^{2} \xi .
$$

Let us focus on the case $\sigma=0$ (symmetric case). For this particular value of the parameter $\sigma$, (10) becomes the famous Wigner function [1]:

$$
W_{A}^{(0)}(q, p)=\int_{-\infty}^{+\infty} \mathrm{e}^{\mathrm{i} p u / \hbar}\left\langle q-\frac{1}{2} u|A| q+\frac{1}{2} u\right\rangle \mathrm{d} u .
$$

The $\star$-product is the Moyal product, whose closed form was introduced by Groenewold in the forties [2]:

$$
W_{A B}^{(0)}(q, p)=\left.W_{A}^{(0)} \mathrm{e}^{-\mathrm{i} \hbar \Gamma / 2} W_{B}^{(0)}\right|_{(q, p)}, \quad \Gamma:=\frac{\overleftarrow{\partial}}{\partial p} \frac{\vec{\partial}}{\partial q}-\frac{\overleftarrow{\partial}}{\partial q} \frac{\vec{\partial}}{\partial p} .
$$

A useful representation of the star product was introduced by Bopp [20]. He found that, for any two operators $A(\hat{q}, \hat{p})$ and $B(\hat{q}, \hat{p})$, the $\star$-product can be cast in the following form:

$$
\begin{aligned}
& W_{A}^{(0)} \star W_{B}^{(0)}=A(Q, P) W_{B}^{(0)} \\
& Q:=q+\frac{\mathrm{i} \hbar}{2} \frac{\partial}{\partial p}, \quad P:=p-\frac{\mathrm{i} \hbar}{2} \frac{\partial}{\partial q}
\end{aligned}
$$


where now $A(Q, P)$ should be understood as an operator acting on $C^{\infty}(\mathcal{P})$. The operators $Q$ and $P$ are usually known as Bopp operators. Observe that $[Q, P]=\mathrm{i} \hbar$, which can be checked directly from (13). As a consequence, $Q$ and $P$ are operators on $C^{\infty}(\mathcal{P})$ satisfying the canonical commutation relations.

Using the reality condition of the SW kernel, we can write the time evolution of the symbol of the density matrix, $W_{\varrho}^{(0)}$, in a simple way:

$$
\partial_{t} W_{\varrho}^{(0)}=\frac{2}{\hbar} \operatorname{Im}(H(Q, P)) W_{\varrho}^{(0)}
$$

with $H$ the Hamiltonian of the system. The classical limit is very easily obtained now. Expanding up to first order in $\hbar$,

$$
H(Q, P)=H(p, q)+\frac{\mathrm{i} \hbar}{2}\left(\partial_{q} H \frac{\partial}{\partial p}-\partial_{p} H \frac{\partial}{\partial q}\right)+O\left(\hbar^{2}\right)
$$

we recover the classical Liouville equation:

$$
\partial_{t} W_{\varrho}^{(0)}=\left\{H, W_{\varrho}^{(0)}\right\} .
$$

\section{The spin case}

We now turn to spin systems, our main interest in this work. The dynamical symmetry group is $S U(2)$, and the phase space is the unit sphere $S^{2}$. The classical Poisson bracket is suitably written in terms of the components of

$$
\mathbf{m}(\theta, \phi)=(\sin \theta \cos \phi, \sin \theta \sin \phi, \cos \theta) .
$$

Namely ${ }^{4}$,

$$
\left\{m_{i}, m_{j}\right\}=\frac{1}{S} \epsilon_{i j k} m_{k},
$$

where $\epsilon_{i j k}$ is the Levi-Civita symbol. Strictly, the Poisson bracket should be $\left\{m_{i}, m_{j}\right\}=$ $g \epsilon_{i j k} m_{k}$, where $g$ is the gyromagnetic ratio, which is related to the quantum spin number by $S=\mu_{B} / g \hbar$, with $\mu_{B}$ is the magnetic moment. From now on we set $\hbar=\mu_{B}=1$, so that $S=1 / g$. The classical Liouville equation may be expressed ([26]) as

$\partial_{t} W_{\varrho}^{(\sigma)}=\left\{H, W_{\varrho}^{(\sigma)}\right\}=-\frac{1}{S} \frac{\partial}{\partial \mathbf{m}} \cdot\left(\mathbf{m} \times \mathbf{B}_{\text {eff }}\right) W_{\varrho}^{(\sigma)}, \quad \mathbf{B}_{\text {eff }}:=-\frac{\partial H}{\partial \mathbf{m}}$,

with the divergence $(\partial / \partial \mathbf{m}) \cdot \mathbf{A}=\sum_{i} \partial A_{i} / \partial m_{i}$. The purpose of this section is to generalize the Bopp operators for the spin case, which simplifies the study of the dynamics in phase-space reported in the literature [28].

\subsection{The SW kernel and the star product}

The SW kernel reads $[12,14,17,18]$ :

$$
\Delta^{(\sigma)}(\theta, \phi)=\sqrt{\frac{4 \pi}{2 S+1}} \sum_{l=0}^{2 S}\langle S, S ; l, 0 \mid S, S\rangle^{-\sigma} \sum_{m=-l}^{l} T_{l m} Y_{l m}^{*}(\theta, \phi)
$$

where $\langle j, j ; l, 0 \mid j, j\rangle$ are Clebsch-Gordan coefficients, $T_{l m}=\sqrt{(2 l+1) /(2 S+1)} \times$ $\sum_{j, j^{\prime}}\left\langle S, j ; S, j^{\prime} \mid S, j^{\prime}\right\rangle\left|S, j^{\prime}\right\rangle\langle S, j|$ are the irreducible tensor operators and $Y_{l m}$ are the spherical harmonics.

\footnotetext{
4 Summation over repeated indices must be understood throughout the paper.
} 
Recently, Klimov and Spinoza derived a differential form for the star product in the spin case [19]:

$W_{A}^{(\sigma)} \star W_{B}^{(\sigma)}=N_{S} \sum_{j} a_{j} \widetilde{F}^{\sigma-1}\left(\mathcal{L}^{2}\right)\left[\left(S^{+(j)} \widetilde{F}^{1-\sigma}\left(\mathcal{L}^{2}\right) W_{A}^{(\sigma)}\right) \otimes\left(S^{-(j)} \widetilde{F}^{1-\sigma}\left(\mathcal{L}^{2}\right) W_{B}^{(\sigma)}\right)\right]$

where $\mathcal{L}^{2}$ is the Casimir operator on the sphere,

$$
\begin{array}{ll}
\mathcal{L}^{2}=-\left[\frac{\partial^{2}}{\partial \theta^{2}}+\cot \theta \frac{\partial}{\partial \theta}+\frac{1}{\sin ^{2} \theta} \frac{\partial^{2}}{\partial \phi^{2}}\right], & \mathcal{L}^{2} Y_{l m}=l(l+1) Y_{l m}, \\
\widetilde{F}\left(\mathcal{L}^{2}\right) Y_{l m}=F(l) Y_{l m}, & F(l)=\sqrt{(2 S+l+1) !(2 S-l) !}, \\
S^{ \pm(j)}=\prod_{k=0}^{j-1}\left(k \cot \theta-\frac{\partial}{\partial \theta} \mp \frac{\mathrm{i}}{\sin \theta} \frac{\partial}{\partial \phi}\right), & a_{j}=\frac{(-1)^{j}}{j !(2 S+j+1) !}, \\
\text { and } N_{S}=\sqrt{2 S+1} F^{\sigma}(0) . &
\end{array}
$$

\subsection{Bopp operators in the spin case}

The evaluation of (21) seems quite involved. Here, we show that it can be simplified by generalizing the Bopp operators (13) for the spin case. That is, we look for operators $\mathcal{S}_{i}^{(\sigma)}$ acting on $C^{\infty}(\mathcal{P})$ such that

$$
W_{S_{i}}^{(\sigma)} \star W_{A}^{(\sigma)}=\mathcal{S}_{i}^{(\sigma)} W_{A}^{(\sigma)}, \quad i=1,2,3 .
$$

Let us invoke the expression derived in the appendix for $W_{S_{3}}^{(\sigma)} \star Y_{l m}$, equation (A.13). Noticing that $m_{3}=\cos \theta, L_{3}=-\mathrm{i} \partial_{\phi}, \mathrm{i}(\mathbf{m} \times \mathbf{L})_{3}=\sin \theta \partial_{\theta}$ and recalling that every function on the sphere is a linear combination of spherical harmonics we deduce that for any operator $A$ :

$$
W_{S_{3}}^{(\sigma)} \star W_{A}^{(\sigma)}=\left[m_{3} \tilde{\eta}_{1}^{(\sigma)}\left(\mathcal{L}^{2}\right)+\mathrm{i}(\mathbf{m} \times \mathbf{L})_{3} \tilde{\eta}_{2}^{(\sigma)}\left(\mathcal{L}^{2}\right)+\frac{1}{2} L_{3}\right] W_{A}^{(\sigma)} .
$$

where $\tilde{\eta}_{i}^{(\sigma)}\left(\mathcal{L}^{2}\right) Y_{l m}=\eta_{i}^{(\sigma)}(l) Y_{l m}, i=1,2$ and

$$
\begin{aligned}
\eta_{1}^{(\sigma)}(l) & =\frac{F^{1-\sigma}(l)}{2(2 l+1)}\left[F^{\sigma-1}(l+1)(2 S+l+2)(l+1)-F^{\sigma-1}(l-1)(l-2 S-1) l\right] \\
\eta_{2}^{(\sigma)}(l) & =\frac{F^{1-\sigma}(l)}{2(2 l+1)}\left[F^{\sigma-1}(l+1)(2 S+l+2)+F^{\sigma-1}(l-1)(l-2 S-1)\right] .
\end{aligned}
$$

The vector $\mathbf{m}$ has been defined in (17) and $\mathbf{L}$ is the angular momentum operator acting on the sphere. Explicitly,

$$
\mathbf{L}=-\mathrm{i}\left(\mathbf{m} \times \frac{\partial}{\partial \mathbf{m}}\right)
$$

which satisfies $\left[L_{i}, L_{j}\right]=\mathrm{i} \epsilon_{i j k} L_{k}$. The following identities are useful:

$$
\left[m_{i}, L_{j}\right]=\mathrm{i} \epsilon_{i j k} m_{k} ; \quad\left[(\mathbf{m} \times \mathbf{L})_{i}, L_{j}\right]=\mathrm{i} \epsilon_{i j k}(\mathbf{m} \times \mathbf{L})_{k} .
$$

Hence,

$$
\mathcal{S}_{3}^{(\sigma)}=m_{3} \tilde{\eta}_{1}^{(\sigma)}\left(\mathcal{L}^{2}\right)+\mathrm{i}(\mathbf{m} \times \mathbf{L})_{3} \tilde{\eta}_{2}^{(\sigma)}\left(\mathcal{L}^{2}\right)+\frac{1}{2} L_{3}
$$

The covariance of the star product at the infinitesimal level implies that

$$
W_{\mathrm{i} \epsilon_{i j k} S_{k} A}^{(\sigma)}=W_{\left[S_{i}, S_{j}\right] A}^{(\sigma)}=\left[L_{i}, \mathcal{S}_{j}^{(\sigma)}\right] W_{A}^{(\sigma)}
$$


whence

$$
\left[\mathcal{S}_{i}^{(\sigma)}, L_{j}\right]=\mathrm{i} \epsilon_{i j k} \mathcal{S}_{k}^{(\sigma)}
$$

Using equation (31) along with (28) and (29) we obtain

$$
\mathcal{S}_{i}^{(\sigma)}=m_{i} \tilde{\eta}_{1}^{(\sigma)}\left(\mathcal{L}^{2}\right)+\mathrm{i}(\mathbf{m} \times \mathbf{L})_{i} \tilde{\eta}_{2}^{(\sigma)}\left(\mathcal{L}^{2}\right)+\frac{1}{2} L_{i}
$$

The operators $\mathcal{S}_{i}^{(\sigma)}$ are the sought generalization of the Bopp operators to the spin case. Now, by the associativity of the star product, we can extend (25) to any function $A\left(S_{1}, S_{2}, S_{3}\right)$ of the spin operators:

$$
W_{A B}^{(\sigma)}=A\left(\mathcal{S}_{1}^{(\sigma)}, \mathcal{S}_{2}^{(\sigma)}, \mathcal{S}_{3}^{(\sigma)}\right) W_{B}^{(\sigma)}
$$

where, equivalently to the spinless particle case (see equation (13)), $A\left(\mathcal{S}_{1}^{(\sigma)}, \mathcal{S}_{2}^{(\sigma)}, \mathcal{S}_{3}^{(\sigma)}\right.$ ) is the same function of its arguments as $A\left(S_{1}, S_{2}, S_{3}\right)$, now acting on $C^{\infty}(\mathcal{P})$. In order to ease the notation we will simply write $A\left(\mathcal{S}^{(\sigma)}\right)$ instead of $A\left(\mathcal{S}_{1}^{(\sigma)}, \mathcal{S}_{2}^{(\sigma)}, \mathcal{S}_{3}^{(\sigma)}\right)$.

The von Neumann dynamics is governed by the equation (compare with (14)):

$$
\partial_{t} W_{\varrho}^{(\sigma)}=2 \operatorname{Im}\left(H\left(\mathcal{S}^{(\sigma)}\right)\right) W_{\varrho}^{(\sigma)} .
$$

Besides $\left[\mathcal{S}_{i}^{(\sigma)}, \mathcal{S}_{j}^{(\sigma)}\right]=\mathrm{i} \epsilon_{i j k} \mathcal{S}_{k}^{(\sigma)}$. In this sense Bopp operators are the phase-space analogues of the corresponding Hilbert-space operators.

Remark. In the $\sigma=0, \pm 1$ cases we can write

$$
\begin{gathered}
\eta_{1}^{(\sigma)}=\left\{\begin{array}{cc}
S & \sigma=-1 \\
\frac{1}{2(2 l+1)}\left[(l+1) \sqrt{(2 S+1)^{2}-(l+1)^{2}}+l \sqrt{(2 S+1)^{2}+l^{2}}\right] & \sigma=0 \\
S+1 & \sigma=1
\end{array}\right. \\
\eta_{2}^{(\sigma)}=\left\{\begin{array}{cc}
-\frac{1}{2} & \sigma=0 \\
\frac{1}{2(2 l+1)}\left[\sqrt{(2 S+1)^{2}-(l+1)^{2}}-\sqrt{(2 S+1)^{2}+l^{2}}\right] & \sigma=1 . \\
\frac{1}{2} & \sigma
\end{array}\right.
\end{gathered}
$$

The form of $\mathcal{S}_{i}^{( \pm 1)}$ was already found by Takahashi and Shibata in [11] by extending the construction of Cahill and Glauber. We have recovered their result and generalized it to any value of $\sigma$ in terms of the symbol (2) for the differential form of the star product of Klimov and Espinoza (21). This covers the important case of symmetric ordering.

\subsection{Large $S$}

We turn to the large- $S$ (or classical) limit. Firstly, note that for $S \gg 1$ we can write [19]

$$
F(l)=\frac{(2 S+1) !}{\sqrt{2 S+1}}\left[1+\frac{1}{2(2 S+1)} l(l+1)+\mathrm{O}\left(\frac{1}{S^{2}}\right)\right] .
$$

Inserting the above expansion in (27) we immediately find that

$$
\begin{aligned}
& 2 \tilde{\eta}_{1}^{(\sigma)}=2 S+1+\sigma+\frac{1}{2(2 S+1)}\left(\mathcal{L}^{2}+1\right)\left(\sigma^{2}-1\right)+\mathrm{O}\left(\frac{1}{S^{2}}\right), \\
& 2 \tilde{\eta}_{2}^{(\sigma)}=\sigma+\frac{1}{2(2 S+1)}\left(\sigma^{2}-1\right)+\mathrm{O}\left(\frac{1}{S^{2}}\right) .
\end{aligned}
$$


The above expressions allow us to evaluate the quasiclassical evolution of $W_{\varrho}^{(\sigma)}$ in a simple way (see also [29]). This is an alternative to the spin coherent path integral formalism used in $[30,31]$. In the classical limit, $\tilde{\eta}_{1}^{(\sigma)}=S+(\sigma+1) / 2+\mathrm{O}(1 / S)$ and $\tilde{\eta}_{2}^{(\sigma)}=\sigma / 2+\mathrm{O}(1 / S)$. Let $H\left(S_{1}, S_{2}, S_{3}\right)$ be the Hamiltonian. Also in the classical limit,

$$
\operatorname{Im}\left(H\left(\mathcal{S}^{(\sigma)}\right)\right)=\frac{-\mathrm{i}}{2 S} \frac{\partial H}{\partial m_{i}} L_{i}
$$

and using that for any $\mathbf{b} \in \mathbb{R}^{3}$,

$$
-\mathbf{i b} \cdot \mathbf{L}=\frac{\partial}{\partial \mathbf{m}} \cdot[\mathbf{m} \times \mathbf{b}],
$$

we recover the classical Poisson bracket (19), obviously independent of $\sigma$.

\subsection{Quadratic Hamiltonians}

We apply now the formalism to the following case:

$$
H=-D_{i j} S_{i} S_{j}-B_{i} S_{i}, \quad D_{i j}=D_{j i}
$$

which is the most general Hamiltonian quadratic in the variables $S_{i}$. This example covers many applications in magnetism [32] and quantum optics (see, e.g., [33, 34]). With the help of (34) and (25) we obtain that $\operatorname{Im}(H)=-\mathrm{i}\left[D_{j k} L_{j}\left(m_{k} \tilde{\eta}_{1}^{(\sigma)}+\mathrm{i}(\mathbf{m} \times \mathbf{L})_{k} \tilde{\eta}_{2}^{(\sigma)}\right)+B_{j} L_{j} / 2\right]$ and using (40) we finally get

$$
\partial_{t} W_{\varrho}^{(\sigma)}=-\frac{1}{S} \frac{\partial}{\partial \mathbf{m}} \cdot\left[\mathbf{m} \times \mathbf{B}_{\mathrm{eff}}^{Q}\right] W_{\varrho}^{(\sigma)}
$$

with

$$
\left[\mathbf{B}_{\mathrm{eff}}^{Q}\right]_{j}=\left[\mathbf{B}_{\mathrm{eff}}\right]_{j}+2 S D_{j k}\left[m_{k}\left(\tilde{\eta}_{1}^{(\sigma)}-S\right)+\mathrm{i}(\mathbf{m} \times \mathbf{L})_{k} \tilde{\eta}_{2}^{(\sigma)}\right] .
$$

Equation (42) is a compact way of writing the quantum dynamics in phase space. $\mathbf{B}_{\mathrm{eff}}^{Q}$ is defined in such a way that the first term gives the classical effective field $\left[\mathbf{B}_{\mathrm{eff}}\right]_{j}=S B_{j}+2 S^{2} D_{j k} m_{k}$ (see equation (19)) and the second one the quantum contributions. When $D_{j k}=0$ the quantum time evolution equation (42) coincides with the classical one [14, Theorem 5]. This is analogous to the harmonic oscillator for the spinless particle [7] where the classical and quantum equation of motion in phase space are the same. Note, however, that although the phase-space time evolution equations in the classical and quantum cases are formally identical, the allowed solutions for the differential equations are different at the classical and quantum levels.

Remark. Consider the particular case $H=-D S_{3}^{2}$. Then, equation (42) simply reads

$$
\partial_{t} W_{\varrho}^{(\sigma)}=-2 D\left(\cos \theta \tilde{\eta}_{1}^{(\sigma)}+\sin \theta \frac{\partial}{\partial \theta} \tilde{\eta}_{2}^{(\sigma)}\right) \frac{\partial W_{\varrho}^{(\sigma)}}{\partial \phi}
$$

with $\tilde{\eta}_{1}^{(\sigma)}$ and $\tilde{\eta}_{2}^{(\sigma)}$ given in (27). For $\sigma= \pm 1$ we recover the results found by Klimov (see equations (65) and (67) of [28]). However, we obtain a different result for $\sigma=0$ (equation (66) in [28]).

Finally, using the asymptotic forms of $\tilde{\eta}_{1}^{(\sigma)}$ and $\tilde{\eta}_{2}^{(\sigma)}$, equation (38), we obtain the quasiclassical form of (42). In the case $H=-D S_{3}^{2}$ the result agrees with derivations previously appeared in the literature (see the conclusions of [29]). 


\section{Application to quantum master equations}

The field of open quantum systems deals with the unavoidable interaction between the system of interest and the environment [21]. Under assumptions such as weak coupling between the environment and the system and fast bath dynamics [25,35], the environment may be modelled as a collection of oscillators. With these hypotheses one can write the total Hamiltonian (system plus environment) as

$$
H_{\mathrm{tot}}=H_{\mathrm{s}}+\sum_{\alpha} \frac{1}{2}\left\{P_{\alpha}^{2}+\omega_{\alpha}^{2}\left[Q_{\alpha}+\frac{c_{\alpha}}{\omega_{\alpha}^{2}} F\right]^{2}\right\}
$$

where $\alpha$ is an oscillator index and the coupling terms $F$ are functions of the system variables.

Classically the dynamics of the system degrees of freedom is formulated in terms of Langevin or Fokker-Planck equations [22,36]. At the quantum level, $Q_{\alpha}$ and $P_{\alpha}$ are the position and momentum operators and $F$ is a Hermitian operator which depends on the system variables. The total system is unlikely to be in a pure state and a density-matrix description is required. For observables depending only on the system variables, the required object is the reduced density operator $\varrho=\operatorname{Tr}_{\text {bath }}\left(\varrho_{\text {tot }}\right)$, where one traces the bath variables out. For weak system-bath coupling a closed dynamical equation for $\varrho$ can be obtained by perturbation theory. This is the case of many problems in quantum optics, chemical physics or magnetism [21]. As far as the classical limit is concerned, a semiclassical quantum master equation would be sufficient. Let $T$ be the temperature of the bath and $\gamma$ the damping coefficient (measuring the coupling strength). Considering that $\gamma /(S T) \ll 1$ one arrives to [24, 23]

$$
\partial_{t} \varrho=-\mathrm{i}\left[H_{\mathrm{s}}, \varrho\right]-\gamma T\left([F, F \varrho]-\frac{1}{2 T}\left[F,\left[H_{\mathrm{s}}, F\right] \varrho\right]+\text { h.c. }\right)
$$

where h.c. means 'Hermitian conjugate'. The term $-\mathrm{i}\left[H_{\mathrm{s}}, \varrho\right]$ gives the isolated-spin unitary evolution and the rest encodes the bath influence.

Now, we address the problem of working out the phase-space transform of (46). In the spinless case for the generic Hamiltonian $H_{\mathrm{s}}=p^{2} / 2 m+V(q)$ and bilinear coupling, $F=q$, the master equation (46) is the celebrated Caldeira-Leggett equation [24]. Then the phasespace transformation gives the first quantum corrections to the Klein-Kramers equation, which is nothing but the Fokker-Planck equation for a particle in a potential [36].

In order to obtain the quantum corrections to the classical Fokker-Planck equations for spins, we will make use of the generalized Bopp operators. Using the results of section 3.2 for transforming Hilbert space operators into operators acting on $C^{\infty}(\mathcal{P})$ we obtain

$$
[F, F \varrho]+\text { h.c. } \quad \mapsto \quad\left(F\left(\mathcal{S}^{(\sigma)}\right)-\text { c.c. }\right)^{2} W_{\varrho}^{(\sigma)}
$$

and

$\left[F,\left[H_{\mathrm{s}}, F\right] \varrho\right]+$ h.c. $\quad \mapsto \quad\left(F\left(\mathcal{S}^{(\sigma)}\right)-\right.$ c.c. $)\left(\left[H_{\mathrm{s}}\left(\mathcal{S}^{(\sigma)}\right), F\left(\mathcal{S}^{(\sigma)}\right)\right]-\right.$ c.c. $) W_{\varrho}^{(\sigma)}$

where c.c. means 'complex conjugate'. Then, equation (46) reads in the phase-space formulation:

$$
\begin{aligned}
\partial_{t} W_{\varrho}^{(\sigma)}=\left[2 \operatorname{Im}\left(H_{\mathrm{s}}\left(\mathcal{S}^{(\sigma)}\right)\right)+4 \gamma T\left[\operatorname{Im}^{2}\left(F\left(\mathcal{S}^{(\sigma)}\right)\right)\right.\right. \\
\left.\left.\quad-\frac{1}{2 T} \operatorname{Im}\left(F\left(\mathcal{S}^{(\sigma)}\right)\right) \operatorname{Im}\left(\left[H_{\mathrm{s}}\left(\mathcal{S}^{(\sigma)}\right), F\left(\mathcal{S}^{(\sigma)}\right)\right]\right)\right]\right] W_{\varrho}^{(\sigma)} .
\end{aligned}
$$

This is the phase-space equivalent of the master equation (46) and should be viewed as the quantum generalization of the classical Fokker-Planck equations. We emphasize that the transformation to the phase-space formalism has been rather simple thanks to (34). 


\subsection{The open isotropic spin versus the open harmonic oscillator}

To gain some insight we particularize equation (49) to an specific example. Our intention here is to highlight the differences between the spinless particle and the spin system. For that, we closely follow the work of Caldeira and Leggett on the quantum master equation for a spinless particle [24]. In this case the phase-space transform of the last two terms of (46) gives the dissipation and diffusion terms of the classical Fokker-Planck equation [25]. In particular, for the harmonic oscillator, both the Caldeira-Leggett and Fokker-Planck equations are identical (see the discussion of section 3.4).

Similarly, in the spin case we also choose a bilineal coupling, $F=\xi_{j} S_{j}$, with $\xi_{j}$ some real constants. For spin systems $\left[H_{\mathrm{s}}, F\right]$ is in general $H_{\mathrm{s}}$-dependent and a generic form of the time evolution equation cannot be given. For the sake of simplicity we take $H_{\mathrm{s}}=-B_{i} S_{i}$, i.e. the isotropic spin $\left(D_{i j}=0\right.$ in (42)). This is the closest analogue to the open quantum oscillator discussed above (section 3.4). Then, the quantum master equation (46) follows in phase-space from (49):

$\partial_{t} W_{\varrho}^{(\sigma)}=-\frac{1}{S} \frac{\partial}{\partial \mathbf{m}} \cdot\left\{\left(\mathbf{m} \times \mathbf{B}_{\mathrm{eff}}\right)-\mathbf{m} \times \widehat{\Lambda}\left[\mathbf{m} \times\left(\mathbf{B}_{\mathrm{eff}}-T \frac{\partial}{\partial \mathbf{m}}\right)+\mathbf{M} \times \mathbf{B}_{\mathrm{eff}}\right]\right\} W_{\varrho}^{(\sigma)}$

where $\left[\mathbf{B}_{\mathrm{eff}}\right]_{j}=S B_{j}$ is the classical effective field (see equation (19)), $\widehat{\Lambda}$ has components

$$
\widehat{\Lambda}_{j k}=\frac{\gamma}{S} \frac{\partial F}{\partial m_{j}} \frac{\partial F}{\partial m_{k}}
$$

(if $F$ is linear, then $\left.\widehat{\Lambda}_{j k}=S \gamma \xi_{j} \xi_{j}\right)$ and $\mathbf{M}=\mathbf{m}\left(\tilde{\eta}_{1}^{(\sigma)}-S\right)+\mathrm{i}(\mathbf{m} \times \mathbf{L}) \tilde{\eta}_{2}^{(\sigma)}$. Observe that $\mathbf{M}=\mathrm{O}(1 / S)$, so the last term vanishes as $S \rightarrow \infty$ recovering the classical Fokker-Planck equation [26].

Hence, even in the case where both system $\left(H_{\mathrm{s}}\right)$ and coupling $(F)$ are linear in the spin variables, classical and quantum Fokker-Planck equations are different, unlike the open harmonic oscillator. Within the phase-space formalism this difference can be worked out explicitly.

\subsection{The classical limit of (49): general case}

To finish, let us go back to (49) and compute its classical limit for general $H_{\mathrm{s}}$ and $F$. Using (39) one finds that

$$
\begin{aligned}
& \operatorname{Im}^{2}\left(F\left(\mathcal{S}^{(\sigma)}\right)\right) \stackrel{S \rightarrow \infty}{\longrightarrow} \frac{1}{4 S^{2}} L_{j} \frac{\partial F}{\partial m_{j}} \frac{\partial F}{\partial m_{k}} L_{k} \\
& \operatorname{Im}\left(\left[H_{\mathrm{S}}\left(\mathcal{S}^{(\sigma)}\right), F\left(\mathcal{S}^{(\sigma)}\right)\right]\right) \stackrel{S \rightarrow \infty}{\longrightarrow}-1 / S\left\{H_{\mathrm{s}}, F\right\} .
\end{aligned}
$$

Hence,

$$
\operatorname{Im}\left(F\left(\mathcal{S}^{(\sigma)}\right)\right) \operatorname{Im}\left(\left[H_{\mathrm{s}}\left(\mathcal{S}^{(\sigma)}\right), F\left(\mathcal{S}^{(\sigma)}\right)\right]\right) \stackrel{S \rightarrow \infty}{\longrightarrow} \frac{\mathrm{i}}{2 S^{2}} L_{j} \frac{\partial F}{\partial m_{j}}\left\{H_{\mathrm{s}}, F\right\}
$$

Since $\left\{H_{\mathrm{s}}, F\right\}=\frac{1}{S} \epsilon_{i j k}\left(\partial_{m_{j}} H_{\mathrm{s}}\right)\left(\partial_{m_{k}} F\right) m_{i}$ we finally obtain

$\partial_{t} W_{\varrho}^{(\sigma)}=-\frac{1}{S} \frac{\partial}{\partial \mathbf{m}} \cdot\left\{\left(\mathbf{m} \times \mathbf{B}_{\mathrm{eff}}\right)-\mathbf{m} \times \widehat{\Lambda}\left[\mathbf{m} \times\left(\mathbf{B}_{\mathrm{eff}}-T \frac{\partial}{\partial \mathbf{m}}\right)\right]\right\} W_{\varrho}^{(\sigma)}$

where $\widehat{\Lambda}_{j k}$ has been defined in (51) and $\mathbf{B}_{\text {eff }}$ in (19). Equation (55) is nothing but the classical Fokker-Planck equation for classical spins (cf Equation (50)) [37] (see also [26]). 


\section{Conclusions}

The phase-space formulation of Quantum Mechanics provides deep insight into the quantumclassical correspondence, mainly due to the fact that the mathematical nature of the observables does not change when going from the classical to the quantum theory: they are always functions on the phase space.

It is well known that for non-relativistic spinless particles Bopp operators simplify the manipulations in the phase-space formalism [20, 7]. In this paper, we have generalized Bopp operators for spin systems and applied them to the problem of the classical limit of spins as well as to open quantum spin systems.

The dynamics of open quantum systems has received a renewed attention due to its role in explaining the emergence of the classical world from quantum mechanics [38]. Open quantum systems are handled with equations for the reduced density matrix, cf Equation (46). They consist of the unitary evolution (von Neumann) and a non-unitary term which incorporates the bath influence. For the spinless case the transformation of the time evolution equations into phase space yields the quantum generalization of the corresponding Fokker-Planck equations. We have accomplished this task in the spin case with the help of the generalized Bopp operators. We have worked out the classical limit $(S \rightarrow \infty)$ of the quantum master equation for the density matrix, and recovered the classical Fokker-Planck equations.

\section{Acknowledgments}

We acknowledge J L García-Palacios and J M Gracia-Bondía for useful discussions. DZ acknowledges financial support through DGA project PRONANOMAG and grant no. B059/2003.

\section{Appendix}

We present here the details for the calculation of equation (25). For that we compute explicitly $W_{S_{3}}^{(\sigma)} \star Y_{l m}$, using (21). We first note that [19]

$$
W_{S_{3}}^{(\sigma)}=\left(\frac{S}{S+1}\right)^{-\sigma / 2} \sqrt{S(S+1)} \cos \theta \sim Y_{1,0} .
$$

Using that $\widetilde{F}\left(\mathcal{L}^{2}\right) \cos \theta=F(1) \cos \theta$ and that in the sum of (21) $j_{\max }=1$, together with

we can write

$$
a_{0}=\frac{1}{(2 S+1) !}, \quad a_{1}=-\frac{a_{0}}{2 S+2}
$$

$W_{S_{3}}^{(\sigma)} \star Y_{l m}=\frac{F^{1-\sigma}(l)}{2}\left(\widetilde{F}^{\sigma-1}\left[\cos \theta Y_{l m}\right]-\frac{1}{2 S+2} \widetilde{F}^{\sigma-1}\left[\left(S^{+(1)} \cos \theta\right)\left(S^{-(1)} Y_{l m}\right)\right]\right)$

here $S^{+(1)}$ acts only over $\cos \theta$, and $S^{-(1)}$ over $Y_{l m}$ with, see equation (24):

$$
S^{ \pm(1)}=-\frac{\partial}{\partial \theta} \mp \frac{\mathrm{i}}{\sin \theta} \frac{\partial}{\partial \phi} \text {. }
$$

Next we make use of the relations:

$$
\cos \theta Y_{l m}=\alpha_{1} Y_{l+1, m}+\alpha_{2} Y_{l-1, m} \quad \sin \theta \frac{\partial}{\partial \theta} Y_{l m}=\beta_{1} Y_{l+1, m}+\beta_{2} Y_{l-1, m}
$$

with

$$
\begin{aligned}
& \alpha_{1}=\sqrt{\frac{(l-m+1)(l+m+1)}{(2 l+1)(2 l+3)}}, \quad \alpha_{2}=\sqrt{\frac{(l-m)(l+m)}{(2 l-1)(2 l+1)}} \\
& \beta_{1}=\alpha_{1} l, \quad \beta_{2}=-\alpha_{2}(l+1) .
\end{aligned}
$$


Manipulating the terms entering (A.3) we find

$F^{1-\sigma}(l) \widetilde{F}^{\sigma-1}\left[\cos \theta Y_{l m}\right]=\alpha_{1} F^{1-\sigma}(l) F^{\sigma-1}(l+1) Y_{l+1 m}+\alpha_{2} F^{1-\sigma}(l) F^{\sigma-1}(l-1) Y_{l-1 m}$

and

$$
\begin{gathered}
F^{1-\sigma}(l) \widetilde{F}^{\sigma-1}\left[\left(S^{+(1)} \cos \theta\right)\left(S^{-(1)} Y_{l m}\right)\right]=-\beta_{1} F^{1-\sigma}(l) F^{\sigma-1}(l+1) Y_{l+1 m} \\
-\beta_{2} F^{1-\sigma}(l) F^{\sigma-1}(l-1) Y_{l-1 m}+\mathrm{i} \frac{\partial}{\partial \phi} Y_{l m} .
\end{gathered}
$$

Now, taking into account that

$$
\begin{aligned}
& Y_{l-1 m}=\frac{1}{(2 l+1) \alpha_{2}}\left(l \cos \theta-\sin \theta \frac{\partial}{\partial \theta}\right) Y_{l m} \\
& Y_{l+1 m}=\frac{1}{(2 l+1) \alpha_{1}}\left((l+1) \cos \theta+\sin \theta \frac{\partial}{\partial \theta}\right) Y_{l m}
\end{aligned}
$$

we get

$$
\begin{gathered}
F^{1-\sigma}(l) \widetilde{F}^{\sigma-1}\left[\cos \theta Y_{l m}\right]=F^{1-\sigma}(l) F^{\sigma-1}(l+1) \frac{1}{2 l+1}\left[(l+1) \cos \theta+\sin \theta \frac{\partial}{\partial \theta}\right] Y_{l m} \\
+F^{1-\sigma}(l) F^{\sigma-1}(l-1) \frac{1}{2 l+1}\left[l \cos \theta-\sin \theta \frac{\partial}{\partial \theta}\right] Y_{l m}
\end{gathered}
$$

and

$$
\begin{aligned}
& F^{1-\sigma}(l) \widetilde{F}^{\sigma-1}\left[\left(S^{+(1)} \cos \theta\right)\left(S^{-(1)} Y_{l m}\right)\right] \\
&=-F^{1-\sigma}(l) F^{\sigma-1}(l+1) \frac{l}{2 l+1}\left[(l+1) \cos \theta+\sin \theta \frac{\partial}{\partial \theta}\right] Y_{l m} \\
&+F^{1-\sigma}(l) F^{\sigma-1}(l-1) \frac{l+1}{2 l+1}\left[(l+1) \cos \theta+\sin \theta \frac{\partial}{\partial \theta}\right] Y_{l m} \\
&+\mathrm{i} \frac{\partial}{\partial \phi} Y_{l m} .
\end{aligned}
$$

Finally, we obtain

$$
W_{S_{3}}^{(\sigma)} \star Y_{l m}=\left[\cos \theta \eta_{1}^{(\sigma)}(l)+\sin \theta \frac{\partial}{\partial \theta} \eta_{2}^{(\sigma)}(l)-\frac{\mathrm{i}}{2} \frac{\partial}{\partial \phi}\right] Y_{l m}
$$

with $\eta_{i}^{(\sigma)}, i=1,2$ defined in (27).

\section{References}

[1] Wigner E 1932 On the quantum correction for thermodynamic equilibrium Phys. Rev. 40 749-59

[2] Groenewold H J 1946 On the principles of elementary quantum mechanics Physica 12405

[3] Moyal J E 1949 Quantum Mechanics as a statistical theory Proc. Camb. Phil. Soc. 4599

[4] Bayen F, Flato M, Frondsal C, Lichnerowicz A and Sternheimer D 1978 Deformation theory and quantization Ann. Phys. 111 61-151

[5] Kontsevich M 2003 Deformation quantization of Poisson manifolds: I Lett. Math. Phys. 66 157-216

[6] Cattaneo A S, Felder G and Tomassini L 2002 From local to global deformation quantization of Poisson manifolds Duke Math. J. 115 329-52

[7] Hillery M, O'Connell R F, Scully M O and Wigner E P 1984 Distribution functions in physics: fundamentals Phys. Rep. 106 121-67

[8] Lee H-W 1995 Theory and application of the quantum phase-space distribution functions Phys. Rep. 259 147-211

[9] Arecchi F T, Courtens E, Gilmore R and Thomas H 1972 Atomic coherent states in quantum optics Phys. Rev. A 62211 
[10] Takahashi Y and Shibata F 1975 Spin coherent state representation in non-equilibrium statistical mechanics J. Phys. Soc. Japan 38656

[11] Takahashi Y and Shibata F 1976 Generalized phase space method in spin systems-spin coherent state representation J. Stat. Phys. 1449

[12] Agarwal G S 1981 Relation between atomic coherent-state representation, state multipoles, and generalized phase-space distributions Phys. Rev. A 242889

[13] Cahill K E and Glauber R J 1969 Density operators and quasiprobability distributions Phys. Rev. 1771882

[14] Várilly J C and Gracia-Bond\{1́\}a J 1989 The Moyal representation for spin Ann. Phys., NY 190 107-48

[15] Figueroa H, Gracia-Bondía J M and Várilly C 1990 Moyal quantization with compact symmetry groups and noncommutative harmonic analysis J. Math. Phys. 31 2664-71

[16] Stratonovich R L 1956 On distributions in representation space Zh. Eksp. Teor. Fiz. 311012 Stratonovich R L 1957 Sov. Phys._JETP 4891

[17] Brif C and Mann A 1999 Phase-space formulation of quantum mechanics and quantum-state reconstruction for physical systems with Lie-group symmetries Phys. Rev. A 59 971-987

[18] Brif C and Mann A 1998 Letter to the Editor: a general theory of phase-space quasiprobability distributions J. Phys. A: Math. Gen. 31 L9-L17

[19] Klimov A B and Espinoza P 2002 Moyal-like form of the star product for generalized SU (2) Stratonovich-Weyl symbols J. Phys. A: Math. Gen. $358435-47$

[20] Bopp F 1956 La mécanique quantique est-elle une mécanique statistique classique particulière? Ann. Inst. H. Poincaré 1581

[21] Weiss U 1993 Quantum Dissipative Systems (Singapore: World Scientific)

[22] Zwanzig R 2001 Nonequilibrium Statistical Mechanics (Oxford: Oxford University Press)

[23] Breuer H-P and Petruccione F 2002 The Theory of Open Quantum Systems (Oxford: Oxford University Press)

[24] Caldeira A O and Leggett A J 1983 Path integral approach to quantum Brownian motion Physica A 121 587-616

[25] Caldeira A O and Leggett A J 1983 Dissipation and quantum tunnelling Ann. Phys. $149374-456$

[26] Garc\{í\}a-Palacios J L 2000 On the statics and dynamics of magneto-anisotropic nanoparticles Adv. Chem. Phys. 112 1-210

[27] Zhang W M, Feng D H and Gilmore R 1990 Coherent states: theory and some applications Rev. Mod. Phys. 62867

[28] Klimov A B 2002 Exact evolution equations for SU (2) quasidistribution functions J. Math. Phys. 43 2202-13

[29] Klimov A B and Espinoza P 2005 Classical evolution of quantum fluctuations in spin-like systems: squeezing and entanglement J. Opt. B: Quantum Semiclass. Opt. 7 183-8

[30] Stone M, Park K-S and Garg A 2000 The semiclassical propagator for spin coherent states J. Math. Phys. 41 8025-49

[31] Garg A, Kochetov E, Park K-S and Stone M 2003 Spin coherent-state path integrals and the instanton calculus J. Math. Phys. 44 48-70

[32] White R M 1983 Quantum Theory of Magnetism 2nd edn (Berlin: Springer)

[33] Agarwal G S, Puri R R and Singh R P 1997 Atomic Schrödinger cat states Phys. Rev. A 56 2249-54

[34] Mølmer K and Sørensen A 1999 Multiparticle entanglement of hot trapped ions Phys. Rev. Lett. 82 1835-8

[35] M\{ö\}hring K and Smilansky U 1980 A semi-classical treatment of dissipative processes based on Feynman's influence functional method Nucl. Phys. A 338 227-68

[36] Risken H 1989 The Fokker-Planck Equation 2nd edn (Berlin: Springer)

[37] Garanin D A, Ishchenko V V and Panina L V 1990 Dynamics of an ensemble of single-domain magnetic particles Theor. Math. Phys. (USSR) 82 169-79

[38] Zurek W H 1991 Decoherence and the transition from quantum to classical Phys. Today 44 36-44 